\title{
Infective endocarditis in pregnancy: Case report and review of the literature
}

\author{
Catherine Connolly', Keelin O'Donoghue', Helen Doran' and \\ Fergus P McCarthy ${ }^{1,2}$
}

\begin{abstract}
Infective endocarditis in pregnancy is associated with high maternal and fetal morbidity and mortality and is estimated to complicate approximately I in 100,000 pregnancies. We report the case of a 33-year-old patient who presented at 30 weeks and 3 days gestation in her third pregnancy. The patient described a 3 week history of feeling generally unwell, an episode of temporary speech disturbance, right shoulder tip pain, left subscapular pain on inspiration and chest discomfort. Investigations included an echocardiogram, which revealed a large mobile mass on the aortic coronary cusp and a small mass on the non-coronary cusp. There was significant aortic regurgitation. Blood cultures were positive for staphylococcus lugdunensis. A diagnosis of infective endocarditis was made. The patient deteriorated, with worsening cardiac function, and proceeded to have a caesarean section on day 7 of admission. Her baby had multiple limb abnormalities, subsequently diagnosed as arthrogryposis multiplex congenita. Aortic valve replacement with a mechanical valve was then performed on day 3 post partum. The patient recovered well post operatively and was discharged home with her baby on day 45 post partum. The commonest complications of IE are congestive cardiac failure, perivalvular extension and systemic embolization. The management of infective endocarditis in pregnancy is similar to that of the non-pregnant however there is high foetal mortality associated with cardiopulmonary by-pass for cardiac surgery. The patient described here developed staphylococcus lugdunensis infective endocarditis, which is a rare but aggressive causative organism in infective endocarditis. Infective endocarditis in pregnancy is a rare but serious condition with significant fetal and maternal morbidity and mortality. Early diagnosis with a multidisciplinary team approach is essential to improve outcomes.
\end{abstract}

\section{Keyword}

Infective endocarditis

\section{Case report}

A 33-year-old patient booked at 12 weeks' gestation in her third pregnancy having had one previous miscarriage and one previous healthy term pregnancy in 2007.

Medical history included a "minor" ventricular septal defect diagnosed in childhood, which had not required surgical intervention. Her last cardiology assessment in 2011 was normal. Body mass index was 36 at booking.

At 30 weeks and 3 days' gestation the patient self-referred to the emergency room with a three-week history of feeling generally unwell, an episode of temporary speech disturbance, right shoulder tip pain, left subscapular pain on inspiration and chest discomfort. She had a sinus tachycardia but was apyrexial. Examination revealed a mild systolic murmur. Laboratory investigations are summarized in Table 1. The working diagnosis was a respiratory tract infection and/or a pulmonary embolus. The patient was admitted, commenced on intravenous cefuroxime $750 \mathrm{mg}$ three times daily and therapeutic dose subcutaneous tinzaparin 175 units/kg once daily. Chest X-ray revealed cardiomegaly with small bilateral pleural effusions. Bedside echocardiogram showed a pericardial effusion of less than $1 \mathrm{~cm}$, left ventricular ejection fraction of greater than $50 \%$ and a hyperdynamic left ventricle. The valves appeared normal. The presumptive diagnosis was changed to pericarditis. However, the patient continued on antibiotics and therapeutic tinzaparin pending the results of CT pulmonary angiography (CTPA).

Obstetric ultrasound at 30 weeks and 5 days and repeated by a fetal medicine specialist reported limited views due to the maternal BMI and a transverse fetal lie.

Growth was on the 39th centile with a normal amniotic fluid index and umbilical artery Doppler studies. Concerns were raised regarding the fetal limbs - the differential diagnosis was of an isolated fetal limb abnormality or skeletal dysplasia. Corticosteroids were administered to promote fetal lung maturity.

On day 5 of admission the patient became acutely unwell with worsening dyspnoea and haemoptysis secondary to pulmonary oedema. Repeat echocardiogram showed a large mobile mass on the aortic coronary cusp and a small mass on the non- coronary cusp. Both the left atrium and ventricle were severely dilated with significant aortic regurgitation. Ejection fraction was greater than $76 \%$ (normal range 55-70\%). Troponin $\mathrm{T}$ was grossly elevated (Table 1). Blood cultures were reported as positive for Gram-positive cocci and the patient was commenced on intravenous teicoplanin (1320 mg once daily) and gentamicin ( $80 \mathrm{mg}$ three times daily). CTPA was negative for pulmonary embolus but showed cardiomegaly, splenomegaly and pulmonary oedema consistent with congestive cardiac failure. A diagnosis of destructive aortic valve endocarditis was made with severe aortic regurgitation and acute cardiac failure. The patient was commenced on a glyceryl trinitrate infusion (GTN) and furosemide 40 mg 4-6 hourly.

Due to the rapidly deteriorating cardiac function, caesarean section (CS) was performed on day 7 of admission in the cardiac operating theatre, in the co-located tertiary referral hospital. The decision for delivery was based on worsening pulmonary oedema secondary to cardiac failure, increasing oxygen requirements (requiring $40 \%$ oxygen to maintain oxygen saturations of $97 \%\left(\mathrm{PO}_{2} 9 \mathrm{kPa}\right.$ on $40 \%$ oxygen $)$ and poor tolerance of GTN infusion secondary to hypotension (100/ $36 \mathrm{mmHg}$ at $1 \mathrm{mg}$ GTN per hour). CS was performed under general anaesthesia, using intra- operative transoesophageal echocardiography to monitor cardiac function. Combined CS and valve replacement was considered but it was decided to perform delayed aortic valve

'Anu Research Centre, Department of Obstetrics and Gynaecology, University College Cork, Cork University Maternity Hospital, Wilton, Cork, Ireland

${ }^{2}$ Division of Women's Health, Women's Health Academic Centre KHP, St. Thomas' Hospital, London, UK

\section{Corresponding author:}

Catherine Connolly, Anu Research Centre, Department of Obstetrics and Gynaecology, University College Cork, Cork University Maternity Hospital Cork, Ireland.

Email: cconnol2@tcd.ie 
replacement semi-electively, to facilitate maximal vegetation sterilisation prior to replacement and to allow abdominal wound healing prior to heparinisation necessary for intraoperative cardiac bypass. At CS a B Lynch suture was performed as prophylaxis for post-partum hemorrhage. A live male infant weighing $1.61 \mathrm{~kg}$ was delivered at 31 weeks and 1 day gestation. He was noted to have multiple limb abnormalities, subsequently diagnosed as arthrogryposis multiplex congenita.

Post delivery, the patient was managed in the cardiac intensive therapy unit. She remained intubated for $9 \mathrm{~h}$ post-operatively and received an oxytocin infusion 4.8 units per hour, $20 \mathrm{mg}$ frusemide 6 hourly and intravenous antibiotics. The patient was re-echoed daily postpartum. Three days later, aortic valve replacement was performed. Operative findings included a large vegetation in the central position of the aortic valve. The aortic valve was excised and replaced with a mechanical valve. The vegetation was excised along with a portion of the tricuspid valve.

Echocardiogram on day 2 post-valve replacement showed that the aortic valve was well seated, with no regurgitation. Blood culture report revealed Staphylococcus lugdunensis, sensitive to teicoplanin and flucloxacillin. The patient received intravenous antibiotics until day 42 postvalve replacement. She and her baby were discharged home on day 45 postpartum. At her most recent cardiology review, echocardiogram showed mild to moderate paraventricular aortic regurgitation. The baby is currently undergoing review for congenital right shoulder dislocation, bilateral hip dislocation and contractures of all limbs.

\section{Discussion}

Infective endocarditis (IE) is rare with an incidence of 3-10 episodes per 100,000 person years. $^{2}$ IE in pregnancy is extremely rare with an overall reported incidence of 1 in 100,000 pregnancies. ${ }^{3}$ IE in

Table I. Laboratory investigations. ${ }^{14}$

\begin{tabular}{lll}
\hline Investigation & Value & Reference range \\
\hline Hemoglobin & $9.6 \mathrm{~g} / \mathrm{dL}$ & $9.5-15 \mathrm{~g} / \mathrm{dL}$ \\
White cell count & $10 \times 10^{9} / \mathrm{L}$ & $5.9-16.9 \times 10^{9} / \mathrm{L}$ \\
C-reactive protein & $123 \mathrm{mg} / \mathrm{L}$ & $0.4-8.1 \mathrm{mg} / \mathrm{L}$ \\
Arterial blood gas & $\mathrm{PH} 7.5$ & $7.39-7.45$ \\
& $\mathrm{PO}_{2} 11.2 \mathrm{kPa}$ & $12.26-14.26 \mathrm{kPa}$ \\
& & $(92-107 \mathrm{mmHg})$ \\
& $\mathrm{PCO}_{2} 3.6 \mathrm{kPa}$ & $3.33-4.39 \mathrm{kPa}$ \\
& & $(25 \mathrm{mmHg}-33 \mathrm{mmHg})$ \\
& $85 \mathrm{ng} / \mathrm{L}$ & $>14 \mathrm{ng} / \mathrm{L}$ \\
Troponin T & & \\
\hline
\end{tabular}

pregnancy has a high maternal (up to $33 \%$ ) and fetal mortality (up to $29 \%$ ). ${ }^{4}$ Risk factors for IE include intravenous drug abuse, a prosthetic valve, previous history of IE and congenital heart disease. ${ }^{5}$ The diagnosis of IE is classified according to the Modified Duke Criteria (summarized in Table 2). ${ }^{6}$

The management of IE in pregnancy is similar to that of a nonpregnant patient. The pathogenesis of IE involves the adherence of bacteria to damaged valves, and the organisms most frequently responsible for IE are those that have a high affinity for adherence to damaged endothelium. These include Staphylococcus aureus, Streptococcus species and enterococci, which cumulatively are responsible for $80 \%$ of IE. The patient described here developed S. lugdunensis IE, a rare causative organism of aggressive endocarditis. ${ }^{7}$ However, a recent case report from our unit describes two cases of $S$. lugdunensis isolates from amniotic fluid samples taken at CS. This was part of a larger study on amniotic fluid bacteriology. ${ }^{8}$ S. lugdunensis is a coagulase negative staphylococci and a known skin commensal which colonises the perineum. ${ }^{9}$

A review of IE cases caused by S. lugdunensis from 1988 to 2008 identified 67 cases with a mean age of 54 years. ${ }^{10}$ Eighty one per cent of cases involved native valves and $87 \%$ were left-sided valvular infection. Twelve per cent of cases had multivalvular involvement. Sixty nine per cent received both medical and surgical therapy which compares to a surgical rate for all organisms of $15-52 \% .{ }^{11}$ The overall mortality was $39 \%$ compared to $10-26 \%$ overall in-hospital mortality of IE.

Congestive heart failure is the commonest complication of IE, occurring in $50-60 \%$ of cases. ${ }^{4}$ Other complications include perivalvular extension including abscess formation, fistulation and pseudo aneurysms. ${ }^{12}$ Systemic emboli occurs in $22-50 \%$ of patients with IE.

The indications for surgery in IE include heart failure due to acute valve regurgitation $(58 \%)$, vegetation size $(54 \%)$, persistent sepsis $(39 \%)$ and embolism $(18 \%) .{ }^{13}$ The valve-specific maternal mortality is estimated to be $42 \%$ for the aortic valve, $22 \%$ for mitral valve and $10 \%$ for tricuspid valve. ${ }^{14} \mathrm{~A}$ viable fetus should be delivered if possible prior to surgery, as there is high fetal mortality (up to 15\%) and morbidity associated with cardiopulmonary bypass. ${ }^{3}$

IE in pregnancy is a rare but serious condition with significant fetal and maternal morbidity and mortality. This case report highlights that although the majority of pregnant women are healthy and experience uncomplicated antenatal courses, obstetricians must be alert that rare and serious disease can, and do, occur in pregnancy. Early diagnosis with a multidisciplinary team approach is essential to improve outcomes.

\section{Declaration of conflicting interests}

None declared.

\section{Funding}

None declared.

Table 2. Modified Duke criteria for the diagnosis of infective endocarditis. ${ }^{a}$

\begin{tabular}{|c|c|}
\hline Major criteria & Minor criteria \\
\hline $\begin{array}{l}\text { - Evidence of endocardial involvement: New valvular regurgitation or } \\
\text { echocardiography positive for infective endocarditis (vegetation, } \\
\text { abscess or new partial dehiscence of prosthetic valve) }\end{array}$ & - Fever $>38^{\circ} \mathrm{C}$ \\
\hline $\begin{array}{l}\text { - Blood cultures positive for endocarditis: Positive blood cultures } \\
\text { (two sets more than } 30 \text { min apart) with a typical infective endocar- } \\
\text { ditis organism or persistently positive blood cultures or a single } \\
\text { positive blood culture for Coxiella burnetti (or phase I IgM antibody } \\
\text { titre }>\text { I:800) }\end{array}$ & $\begin{array}{l}\text { - Vascular phenomenon, i.e. major arterial emboli, Janeway lesions } \\
\text { - Immunologic phenomenon, i.e. Osler's nodes, glomerulonephritis } \\
\text { - Microbiological evidence suggestive of infective endocarditis that does } \\
\text { not meet major criteria } \\
\text { - Predisposing cardiac condition or intravenous drug user }\end{array}$ \\
\hline
\end{tabular}

${ }^{a}$ The diagnosis of IE may be made if two or more major criteria are present or one major and three minor criteria or five minor criteria. The diagnosis of IE may be considered in the presence of one major and one minor criterion or three minor criteria. 


\section{Ethical approval}

Written consent from patient for publication.

\section{Guarantor}

Fergus McCarthy.

\section{Contributorship}

None.

\section{References}

1. Moreillon P and Que YA. Infective endocarditis. Lancet 2004; 363: 139-149.

2. Regitz-Zagrosek V, Blomstrom Lundqvist C, Borghi C, et al. ESC Guidelines on the management of cardiovascular diseases during pregnancy: the task force on the management of cardiovascular diseases during pregnancy of the European Society of Cardiology (ESC). Eur Heart $J$ 2011; 32: 3147-3197.

3. Montoya ME, Karnath BM and Ahmad M. Endocarditis during pregnancy. Southern Med J 2003; 96: 1156-1157.

4. Krcmery V, Gogova M, Ondrusova A, et al. Etiology and risk factors of 339 cases of infective endocarditis: report from a 10-year national prospective survey in the Slovak Republic. J Chemotherapy 2003; 15: 579-583.
5. Li JS, Sexton DJ, Mick N, et al. Proposed modifications to the Duke criteria for the diagnosis of infective endocarditis. Clin Inf Dis: Off Publ Infect Dis Soc Am 2000; 30: 633-638.

6. Frank KL, Del Pozo JL and Patel R. From clinical microbiology to infection pathogenesis: how daring to be different works for Staphylococcus lugdunensis. Clin Microbiol Rev 2008; 21: 111-133.

7. Marchocki Z, Collins K, Lehane E, et al. Staphylococcus lugdunensis cultured from the amniotic fluid at Caesarean section. PLoS ONE 2013; 8: e56373.

8. Fleurette J, Bes M, Brun Y, et al. Clinical isolates of Staphylococcus lugdunensis and S. schleiferi: bacteriological characteristics and susceptibility to antimicrobial agents. Res Microbiol 1989; 140: 107-118

9. Liu PY, Huang YF, Tang CW, et al. Staphylococcus lugdunensis infective endocarditis: a literature review and analysis of risk factors. J Microbiol Immunol Infect 2010; 43: 478-484.

10. Sandre RM and Shafran SD. Infective endocarditis: review of 135 cases over 9 years. Clin Infect Dis 1996; 22: 276-286.

11. Carpenter JL. Perivalvular extension of infection in patients with infectious endocarditis. Rev Infect Dis 1991; 13: 127-138.

12. Chambers CE and Clark SL. Cardiac surgery during pregnancy. Clin Obstetr Gynecol 1994; 37: 316-323.

13. Campuzano K, Roque H, Bolnick A, et al. Bacterial endocarditis complicating pregnancy: case report and systematic review of the literature. Arch Gynecol Obstetr 2003; 268: 251-255.

14. Abbassi-Ghanavati $M$, Greer LG and Cunningham FG. Pregnancy and laboratory studies: a reference table for clinicians. Obstetr Gynecol 2009; 114: 1326-1331. 